\title{
Interactions of non-steroidal anti-inflammatory drugs and their bismuth analogues (BiNSAIDs) with biological membrane mimics at physiological pH
}

Tara E. Oliver, Stefania Piantavigna, Philip C. Andrews, Stephen A. Holt and Carolyn T. Dillon*

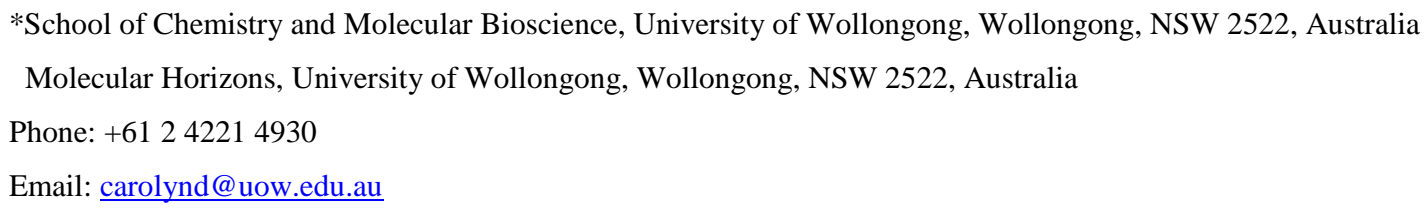

\section{SUPPORTING INFORMATION}

Figure S1. Example conductance traces for a POPC membrane mimic

Table S1. Statistical analysis of the change in membrane conductance of POPC membrane mimics treated with NSAIDs or BiNSAIDs

Table S2. Statistical analysis of the change in membrane conductance of POPC membrane mimics with tolfH and $\mathrm{Bi}(\text { tolf })_{3}$ omitted ....................................... 3

Table S3. Statistical analysis of the change in membrane conductance of POPC/cholesterol membrane mimics treated with NSAIDs or BiNSAIDs ...... 4

Table S4. Statistical analysis of the change in membrane conductance of POPC/cholesterol membrane mimics with tolfH and Bi(tolf) $)_{3}$ omitted ...........5

Figure S2. The fraction of the original bilayer conductance for NSAIDs and BiNSAIDs on POPC membrane mimics, following a $\mathrm{NaCl}$ wash

Figure S3. The fraction of the original bilayer conductance for NSAIDs and BiNSAIDs on POPC/cholesterol membrane mimics, following a $\mathrm{NaCl}$ wash

Table S5. Statistical analysis of the mass deposition onto POPC membrane mimics treated with NSAIDs or BiNSAIDs

Table S6. Statistical analysis of the mass deposition onto POPC/cholesterol membrane mimics treated with NSAIDs or BiNSAIDs

Figure S4. Reflectivity profiles for POPC membrane mimics 10

Figure S5. Reflectivity profiles for d-POPC/cholesterol membrane mimics .11 
A

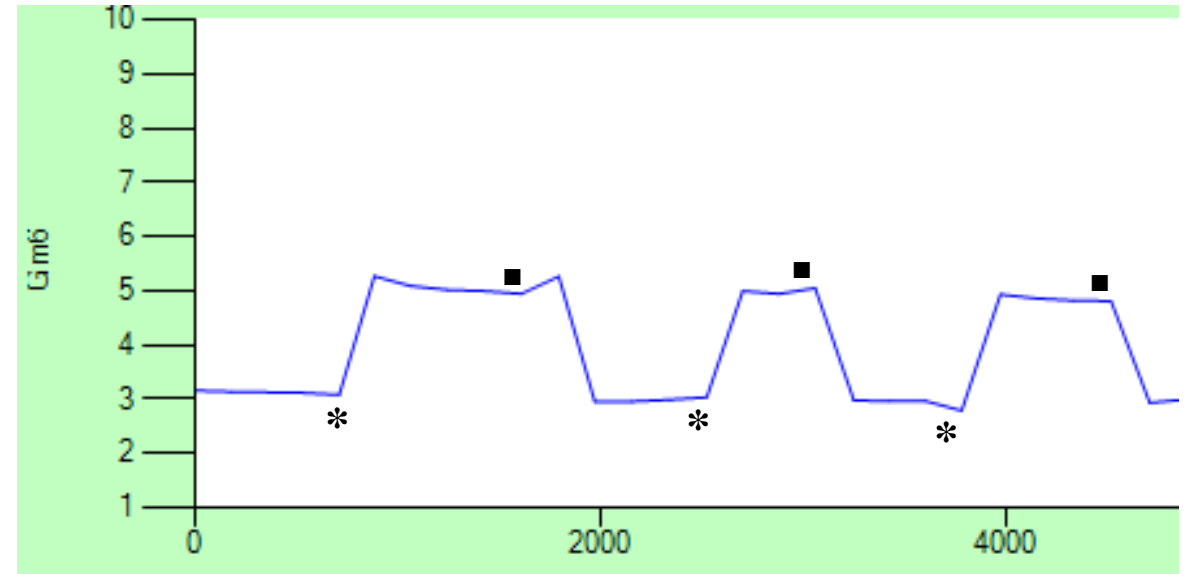

B

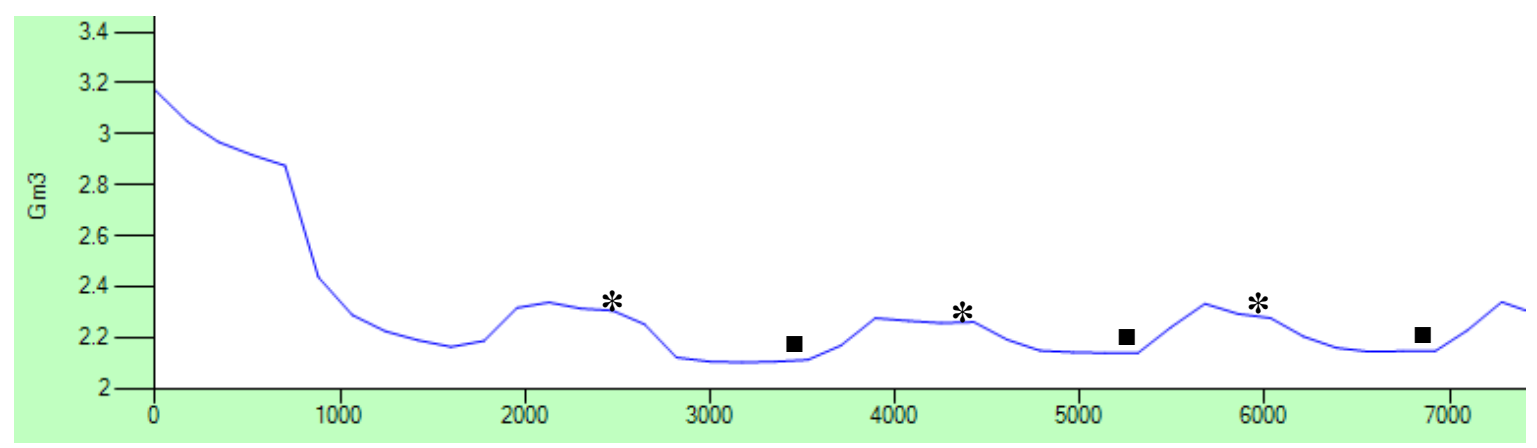

Figure S1. Example EIS traces showing the changes in conductance ( $\mu \mathrm{S}, \mathrm{y}$-axis) of a POPC membrane mimic over time (sec, $\mathrm{x}$-axis). The time points at which compound was introduced to the system are indicated by stars $(*)$ and the time points of the subsequent $\mathrm{NaCl}$ washes are indicated by squares ( $(\mathbf{m})$. Three additions of compound and the subsequent $\mathrm{NaCl}$ washes are shown in each case. (A) The EIS trace for mefH $(480 \mu \mathrm{M})$, which resulted in a positive change in conductance. (B) The EIS trace for $\mathrm{Bi}(\operatorname{asp})_{3}$, which resulted in a negative change in conductance. The initial part of the trace $(0-2000 \mathrm{sec})$ was ignored as the trace was not stable. 
Table S1. Statistical analysis comparing the change in membrane conductance of membrane mimics composed of POPC following treatment with NSAIDs $(480 \mu \mathrm{M})$ or BiNSAIDs $(160 \mu \mathrm{M})$.

\begin{tabular}{|c|c|c|}
\hline $\begin{array}{l}\text { Tukey's Multiple } \\
\text { Comparison Test }\end{array}$ & Significant? & $P<$ \\
\hline TolfH vs. Bi(tolf) $)_{3}$ & Yes & 0.0001 \\
\hline TolfH vs. AspH & Yes & 0.0001 \\
\hline TolfH vs. Bi(asp) $)_{3}$ & Yes & 0.0001 \\
\hline TolfH vs. IndoH & Yes & 0.0001 \\
\hline TolfH vs. Bi(indo) ${ }_{3}$ & Yes & 0.0001 \\
\hline TolfH vs. MefH & Yes & 0.0001 \\
\hline TolfH vs. Bi(mef $)_{3}$ & Yes & 0.0001 \\
\hline Bi(tolf) $)_{3}$ vs. AspH & Yes & 0.0001 \\
\hline $\mathrm{Bi}(\text { tolf })_{3}$ vs. $\mathrm{Bi}(\operatorname{asp})_{3}$ & Yes & 0.0001 \\
\hline $\mathrm{Bi}(\text { tolf })_{3}$ vs. IndoH & Yes & 0.0001 \\
\hline $\mathrm{Bi}(\text { tolf })_{3}$ vs. $\mathrm{Bi}(\text { indo })_{3}$ & Yes & 0.0001 \\
\hline Bi(tolf) $)_{3}$ vs. MefH & Yes & 0.0001 \\
\hline $\mathrm{Bi}(\text { tolf })_{3}$ vs. $\mathrm{Bi}(\text { mef })_{3}$ & Yes & 0.0001 \\
\hline AspH vs. Bi(asp) 3 & No & ns \\
\hline AspH vs. IndoH & No & ns \\
\hline AspH vs. $\mathrm{Bi}(\text { indo })_{3}$ & No & ns \\
\hline AspH vs. MefH & No & ns \\
\hline AspH vs. $\mathrm{Bi}(\mathrm{mef})_{3}$ & No & ns \\
\hline $\mathrm{Bi}(\text { asp) })_{3}$ vs. IndoH & No & ns \\
\hline $\mathrm{Bi}(\operatorname{asp})_{3}$ vs. $\mathrm{Bi}(\text { indo })_{3}$ & No & ns \\
\hline $\mathrm{Bi}(\operatorname{asp})_{3}$ vs. MefH & No & ns \\
\hline $\mathrm{Bi}(\operatorname{asp})_{3}$ vs. $\mathrm{Bi}(\text { mef })_{3}$ & No & ns \\
\hline IndoH vs. $\mathrm{Bi}(\text { indo })_{3}$ & No & ns \\
\hline IndoH vs. MefH & No & ns \\
\hline IndoH vs. $\mathrm{Bi}(\mathrm{mef})_{3}$ & No & ns \\
\hline Bi(indo) $)_{3}$ vs. MefH & No & ns \\
\hline $\mathrm{Bi}(\text { indo })_{3}$ vs. $\mathrm{Bi}(\mathrm{mef})_{3}$ & No & ns \\
\hline MefH vs. $\mathrm{Bi}(\mathrm{mef})_{3}$ & No & ns \\
\hline
\end{tabular}


Table S2. Statistical analysis comparing the change in membrane conductance of membrane mimics composed of POPC following treatment with NSAIDs $(480 \mu \mathrm{M})$ or BiNSAIDs $(160 \mu \mathrm{M})$, omitting the high tolfH and $\mathrm{Bi}\left(\right.$ tolf $_{3}$ values.

\begin{tabular}{|c|c|c|}
\hline $\begin{array}{l}\text { Tukey's Multiple } \\
\text { Comparison Test }\end{array}$ & Significant? & $P<$ \\
\hline AspH vs. $\mathrm{Bi}(\operatorname{asp})_{3}$ & No & ns \\
\hline AspH vs. IndoH & Yes & 0.0001 \\
\hline AspH vs. Bi(indo $)_{3}$ & Yes & 0.0001 \\
\hline AspH vs. MefH & Yes & 0.0001 \\
\hline AspH vs. $\mathrm{Bi}(\mathrm{mef})_{3}$ & Yes & 0.0001 \\
\hline $\mathrm{Bi}(\operatorname{asp})_{3}$ vs. IndoH & Yes & 0.0001 \\
\hline $\mathrm{Bi}(\text { asp })_{3}$ vs. $\mathrm{Bi}(\text { indo })_{3}$ & Yes & 0.0001 \\
\hline $\mathrm{Bi}(\operatorname{asp})_{3}$ vs. MefH & Yes & 0.0001 \\
\hline $\mathrm{Bi}(\operatorname{asp})_{3}$ vs. $\mathrm{Bi}(\text { mef })_{3}$ & Yes & 0.0001 \\
\hline IndoH vs. Bi(indo $)_{3}$ & No & ns \\
\hline IndoH vs. MefH & Yes & 0.0001 \\
\hline IndoH vs. $\mathrm{Bi}(\mathrm{mef})_{3}$ & Yes & 0.0001 \\
\hline $\mathrm{Bi}(\text { indo) })_{3}$ vs. MefH & Yes & 0.0001 \\
\hline $\mathrm{Bi}(\text { indo })_{3}$ vs. $\mathrm{Bi}(\mathrm{mef})_{3}$ & Yes & 0.0001 \\
\hline MefH vs. $\mathrm{Bi}(\mathrm{mef})_{3}$ & No & ns \\
\hline
\end{tabular}


Table S3. Statistical analysis comparing the change in membrane conductance of membrane mimics composed of POPC/cholesterol following treatment with NSAIDs $(480 \mu \mathrm{M})$ or BiNSAIDs $(160 \mu \mathrm{M})$.

\begin{tabular}{|c|c|c|}
\hline $\begin{array}{l}\text { Tukey's Multiple } \\
\text { Comparison Test }\end{array}$ & Significant? & $P<$ \\
\hline TolfH vs. Bi(tolf) $)_{3}$ & Yes & 0.0001 \\
\hline TolfH vs. AspH & Yes & 0.0001 \\
\hline TolfH vs. Bi(asp) 3 & Yes & 0.0001 \\
\hline TolfH vs. IndoH & Yes & 0.0001 \\
\hline TolfH vs. $\mathrm{Bi}(\text { indo })_{3}$ & Yes & 0.0001 \\
\hline TolfH vs. MefH & Yes & 0.0001 \\
\hline TolfH vs. $\mathrm{Bi}(\mathrm{mef})_{3}$ & Yes & 0.0001 \\
\hline $\mathrm{Bi}(\text { tolf })_{3}$ vs. AspH & Yes & 0.0001 \\
\hline $\mathrm{Bi}(\text { tolf })_{3}$ vs. $\mathrm{Bi}(\text { asp })_{3}$ & Yes & 0.0001 \\
\hline $\mathrm{Bi}$ (tolf) $)_{3}$ vs. IndoH & Yes & 0.0001 \\
\hline $\mathrm{Bi}(\text { tolf })_{3}$ vs. $\mathrm{Bi}(\text { indo })_{3}$ & Yes & 0.0001 \\
\hline $\mathrm{Bi}(\text { tolf })_{3}$ vs. MefH & Yes & 0.0001 \\
\hline $\mathrm{Bi}(\text { tolf })_{3}$ vs. $\mathrm{Bi}(\mathrm{mef})_{3}$ & Yes & 0.0001 \\
\hline AspH vs. Bi(asp) $)_{3}$ & No & ns \\
\hline AspH vs. IndoH & No & ns \\
\hline AspH vs. $\mathrm{Bi}$ (indo) $)_{3}$ & No & ns \\
\hline AspH vs. MefH & Yes & 0.05 \\
\hline AspH vs. $\mathrm{Bi}(\mathrm{mef})_{3}$ & Yes & 0.01 \\
\hline $\mathrm{Bi}(\mathrm{asp})_{3}$ vs. IndoH & No & ns \\
\hline $\mathrm{Bi}(\operatorname{asp})_{3}$ vs. $\mathrm{Bi}(\text { indo })_{3}$ & No & ns \\
\hline $\mathrm{Bi}(\operatorname{asp})_{3}$ vs. MefH & Yes & 0.05 \\
\hline $\mathrm{Bi}(\operatorname{asp})_{3}$ vs. $\mathrm{Bi}(\mathrm{mef})_{3}$ & Yes & 0.01 \\
\hline IndoH vs. $\mathrm{Bi}(\text { indo })_{3}$ & No & ns \\
\hline IndoH vs. MefH & No & ns \\
\hline IndoH vs. $\mathrm{Bi}(\mathrm{mef})_{3}$ & No & ns \\
\hline $\mathrm{Bi}(\text { indo })_{3}$ vs. MefH & No & ns \\
\hline $\mathrm{Bi}(\text { indo })_{3}$ vs. $\mathrm{Bi}(\mathrm{mef})_{3}$ & No & ns \\
\hline MefH vs. $\operatorname{Bi}(m e f)_{3}$ & No & ns \\
\hline
\end{tabular}


Table S4. Statistical analysis comparing the change in membrane conductance of membrane mimics composed of POPC/cholesterol following treatment with NSAIDs $(480 \mu \mathrm{M})$ or BiNSAIDs $(160 \mu \mathrm{M})$, omitting the high tolfH and $\mathrm{Bi}(\text { tolf })_{3}$ values.

\begin{tabular}{|c|c|c|}
\hline $\begin{array}{l}\text { Tukey's Multiple } \\
\text { Comparison Test }\end{array}$ & Significant? & $P<$ \\
\hline AspH vs. $\mathrm{Bi}(\operatorname{asp})_{3}$ & No & ns \\
\hline AspH vs. IndoH & Yes & 0.0001 \\
\hline AspH vs. $\mathrm{Bi}(\text { indo })_{3}$ & Yes & 0.0001 \\
\hline AspH vs. MefH & Yes & 0.0001 \\
\hline $\mathrm{AspH}$ vs. $\mathrm{Bi}(\mathrm{mef})_{3}$ & Yes & 0.0001 \\
\hline $\mathrm{Bi}(\operatorname{asp})_{3}$ vs. IndoH & Yes & 0.0001 \\
\hline $\mathrm{Bi}(\text { asp })_{3}$ vs. $\mathrm{Bi}(\text { indo })_{3}$ & Yes & 0.001 \\
\hline $\mathrm{Bi}(\mathrm{asp})_{3}$ vs. MefH & Yes & 0.0001 \\
\hline $\mathrm{Bi}(\operatorname{asp})_{3}$ vs. $\mathrm{Bi}(\mathrm{mef})_{3}$ & Yes & 0.0001 \\
\hline IndoH vs. $\mathrm{Bi}(\text { indo })_{3}$ & No & ns \\
\hline IndoH vs. MefH & Yes & 0.0001 \\
\hline IndoH vs. $\mathrm{Bi}(\mathrm{mef})_{3}$ & Yes & 0.0001 \\
\hline $\mathrm{Bi}(\text { indo) })_{3}$ vs. MefH & Yes & 0.0001 \\
\hline $\mathrm{Bi}(\text { indo })_{3}$ vs. $\mathrm{Bi}(\mathrm{mef})_{3}$ & Yes & 0.0001 \\
\hline MefH vs. $\mathrm{Bi}(\mathrm{mef})_{3}$ & Yes & 0.05 \\
\hline
\end{tabular}




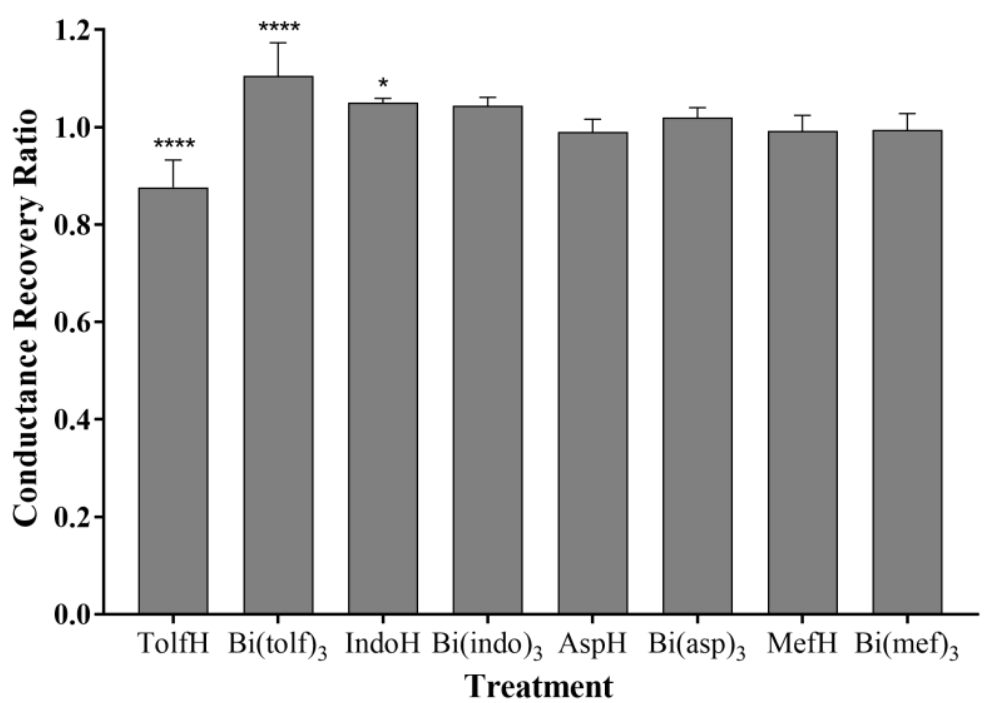

Figure S2. The fraction of the original bilayer conductance for NSAIDs and BiNSAIDs on POPC membrane mimics, following a $\mathrm{NaCl}$ wash, as determined by EIS. The original POPC bilayer was assigned an arbitrary value of 1.0 and the conductance of the system following a $\mathrm{NaCl}$ wash was compared to this value and expressed as a fraction of the original bilayer conductance. Statistical significance in relation to an original bilayer fraction of 1.0 is indicated by $* * * *(P<0.0001)$ or $*(P<0.05)$. 


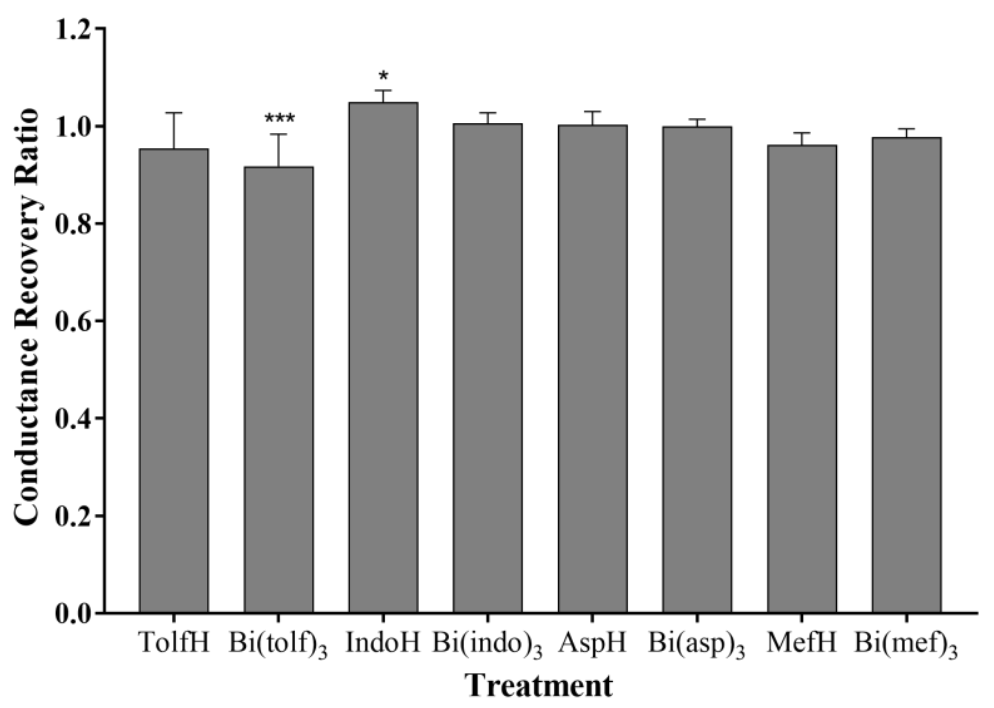

Figure S3. The fraction of the original bilayer conductance for NSAIDs and BiNSAIDs on $\mathrm{POPC} /$ cholesterol membrane mimics, following a $\mathrm{NaCl}$ wash, as determined by EIS. The original POPC/cholesterol bilayer was assigned an arbitrary value of 1.0 and the conductance of the system following a $\mathrm{NaCl}$ wash was compared to this value and expressed as a fraction of the original bilayer conductance. Statistical significance in relation to an original bilayer fraction of 1.0 is indicated by $* * *(P<0.001)$ or $*(P<0.05)$. 
Table S5. Statistical analysis comparing the mass depositions onto membrane mimics composed of POPC following treatment with BiNSAIDs $(160 \mu \mathrm{M})$ or an equivalent concentration (by mass) of free NSAIDs.

\begin{tabular}{|c|c|c|}
\hline $\begin{array}{l}\text { Tukey's Multiple } \\
\text { Comparison Test }\end{array}$ & Significant? & $P<$ \\
\hline TolfH vs. Bi(tolf) $)_{3}$ & Yes & 0.0001 \\
\hline TolfH vs. IndoH & No & ns \\
\hline TolfH vs. $\mathrm{Bi}$ (indo) ${ }_{3}$ & No & ns \\
\hline TolfH vs. AspH & No & ns \\
\hline TolfH vs. Bi(asp) 3 & No & ns \\
\hline TolfH vs. MefH & No & ns \\
\hline TolfH vs. $\mathrm{Bi}(\mathrm{mef})_{3}$ & Yes & 0.0001 \\
\hline $\mathrm{Bi}$ (tolf) $)_{3}$ vs. IndoH & Yes & 0.01 \\
\hline $\mathrm{Bi}(\text { tolf })_{3}$ vs. $\mathrm{Bi}(\text { indo })_{3}$ & Yes & 0.01 \\
\hline $\mathrm{Bi}(\text { tolf })_{3}$ vs. AspH & Yes & 0.01 \\
\hline $\mathrm{Bi}(\text { tolf })_{3}$ vs. $\mathrm{Bi}(\text { asp })_{3}$ & Yes & 0.01 \\
\hline $\mathrm{Bi}(\text { tolf })_{3}$ vs. MefH & Yes & 0.0001 \\
\hline $\mathrm{Bi}(\text { tolf })_{3}$ vs. $\mathrm{Bi}(\mathrm{mef})_{3}$ & Yes & 0.0001 \\
\hline IndoH vs. $\mathrm{Bi}$ (indo) $)_{3}$ & No & ns \\
\hline IndoH vs. AspH & No & ns \\
\hline IndoH vs. $\mathrm{Bi}(\text { asp })_{3}$ & No & ns \\
\hline IndoH vs. MefH & No & ns \\
\hline IndoH vs. $\mathrm{Bi}(\mathrm{mef})_{3}$ & Yes & 0.0001 \\
\hline $\mathrm{Bi}(\text { indo })_{3}$ vs. AspH & No & ns \\
\hline $\mathrm{Bi}(\text { indo })_{3}$ vs. $\mathrm{Bi}(\mathrm{asp})_{3}$ & No & ns \\
\hline $\mathrm{Bi}(\text { indo })_{3}$ vs. MefH & Yes & 0.05 \\
\hline $\mathrm{Bi}(\text { indo })_{3}$ vs. $\mathrm{Bi}(\text { mef })_{3}$ & Yes & 0.0001 \\
\hline AspH vs. Bi(asp) 3 & No & ns \\
\hline AspH vs. MefH & Yes & 0.05 \\
\hline $\mathrm{AspH}$ vs. $\mathrm{Bi}(\mathrm{mef})_{3}$ & Yes & 0.0001 \\
\hline $\mathrm{Bi}(\operatorname{asp})_{3}$ vs. MefH & Yes & 0.01 \\
\hline $\mathrm{Bi}(\operatorname{asp})_{3}$ vs. $\mathrm{Bi}(\text { mef })_{3}$ & Yes & 0.0001 \\
\hline MefH vs. $\operatorname{Bi}(m e f)_{3}$ & Yes & 0.01 \\
\hline
\end{tabular}


Table S6. Statistical analysis comparing the mass depositions onto membrane mimics composed of POPC/cholesterol following treatment with BiNSAIDs $(160 \mu \mathrm{M})$ or an equivalent concentration (by mass) of free NSAIDs.

\begin{tabular}{|c|c|c|}
\hline $\begin{array}{l}\text { Tukey's Multiple } \\
\text { Comparison Test }\end{array}$ & Significant? & $P<$ \\
\hline TolfH vs. Bi(tolf) $)_{3}$ & No & $\mathrm{ns}$ \\
\hline TolfH vs. IndoH & No & $\mathrm{ns}$ \\
\hline TolfH vs. Bi(indo) ${ }_{3}$ & No & ns \\
\hline TolfH vs. AspH & No & ns \\
\hline TolfH vs. Bi(asp) $)_{3}$ & No & ns \\
\hline TolfH vs. MefH & No & ns \\
\hline TolfH vs. $\mathrm{Bi}(\mathrm{mef})_{3}$ & No & ns \\
\hline $\mathrm{Bi}$ (tolf) $)_{3}$ vs. IndoH & No & ns \\
\hline $\mathrm{Bi}(\text { tolf })_{3}$ vs. $\mathrm{Bi}(\text { indo })_{3}$ & No & ns \\
\hline $\mathrm{Bi}(\text { tolf })_{3}$ vs. AspH & No & ns \\
\hline $\mathrm{Bi}(\text { tolf })_{3}$ vs. $\mathrm{Bi}(\text { asp })_{3}$ & No & ns \\
\hline $\mathrm{Bi}(\text { tolf })_{3}$ vs. MefH & No & ns \\
\hline $\mathrm{Bi}(\text { tolf })_{3}$ vs. $\mathrm{Bi}(\mathrm{mef})_{3}$ & No & ns \\
\hline IndoH vs. Bi(indo) ${ }_{3}$ & No & ns \\
\hline IndoH vs. AspH & No & ns \\
\hline IndoH vs. $\mathrm{Bi}(\mathrm{asp})_{3}$ & No & ns \\
\hline IndoH vs. MefH & No & ns \\
\hline IndoH vs. $\mathrm{Bi}(\mathrm{mef})_{3}$ & No & ns \\
\hline $\mathrm{Bi}(\text { indo })_{3}$ vs. $\mathrm{AspH}$ & No & ns \\
\hline $\mathrm{Bi}(\text { indo })_{3}$ vs. $\mathrm{Bi}(\mathrm{asp})_{3}$ & No & ns \\
\hline $\mathrm{Bi}(\text { indo })_{3}$ vs. MefH & No & ns \\
\hline $\mathrm{Bi}(\text { indo })_{3}$ vs. $\mathrm{Bi}(\mathrm{mef})_{3}$ & No & ns \\
\hline AspH vs. $\mathrm{Bi}(\operatorname{asp})_{3}$ & No & ns \\
\hline AspH vs. MefH & No & ns \\
\hline AspH vs. $\mathrm{Bi}(\mathrm{mef})_{3}$ & No & ns \\
\hline $\mathrm{Bi}(\text { asp) })_{3}$ vs. MefH & No & ns \\
\hline $\mathrm{Bi}(\operatorname{asp})_{3}$ vs. $\mathrm{Bi}(\text { mef })_{3}$ & No & ns \\
\hline MefH vs. $\mathrm{Bi}(\mathrm{mef})_{3}$ & No & ns \\
\hline
\end{tabular}



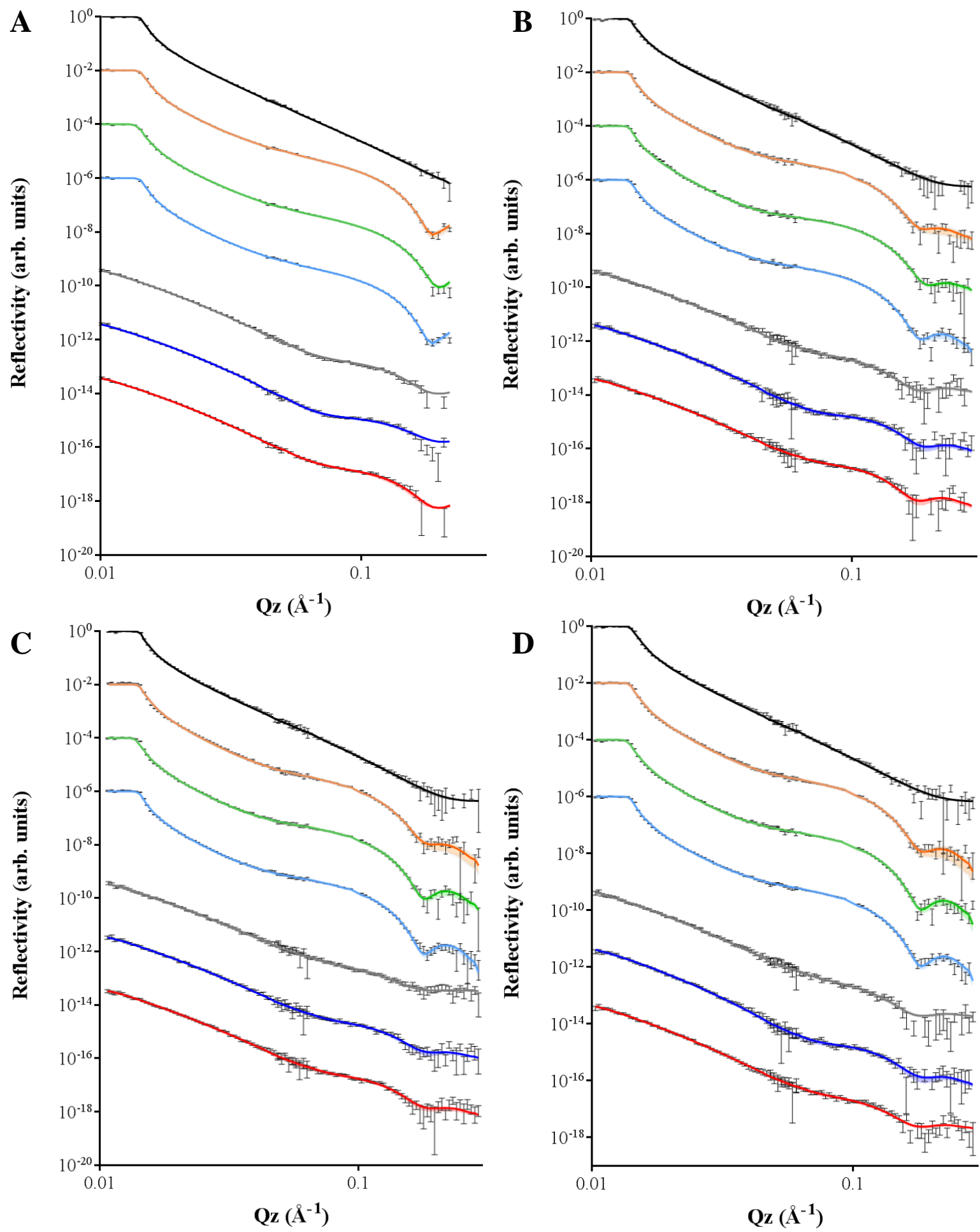

Figure S4. Reflectivity profiles for the treatment of POPC membrane mimics with (A) tolfH $(480 \mu \mathrm{M}),(\mathbf{B}) \mathrm{Bi}(\text { tolf })_{3}(160 \mu \mathrm{M}),(\mathbf{C}) \operatorname{aspH}(480 \mu \mathrm{M})$ or (D) Bi(asp) $3(160 \mu \mathrm{M})$. The profiles are indicative of: plain silicon in $\mathrm{D}_{2} \mathrm{O} / \mathrm{PBS}$ (black); the POPC bilayer in $\mathrm{D}_{2} \mathrm{O} / \mathrm{PBS}$ (orange) and $\mathrm{H}_{2} \mathrm{O} / \mathrm{PBS}$ (grey); the POPC bilayer following treatment with compound in $\mathrm{D}_{2} \mathrm{O} / \mathrm{PBS}$ (green) and $\mathrm{H}_{2} \mathrm{O} / \mathrm{PBS}$ (dark blue) and; the POPC bilayer following a rinse with $\mathrm{D}_{2} \mathrm{O} / \mathrm{PBS}$ (light blue) and $\mathrm{H}_{2} \mathrm{O} / \mathrm{PBS}$ (red). Solid lines represent the best fit obtained from the data analysis and the shaded regions represent the $95 \%$ confidence intervals of the fits. Error bars represent the counting statistics (square root of the number of counts) from both the transmission and data runs propagated through the reduction process. 

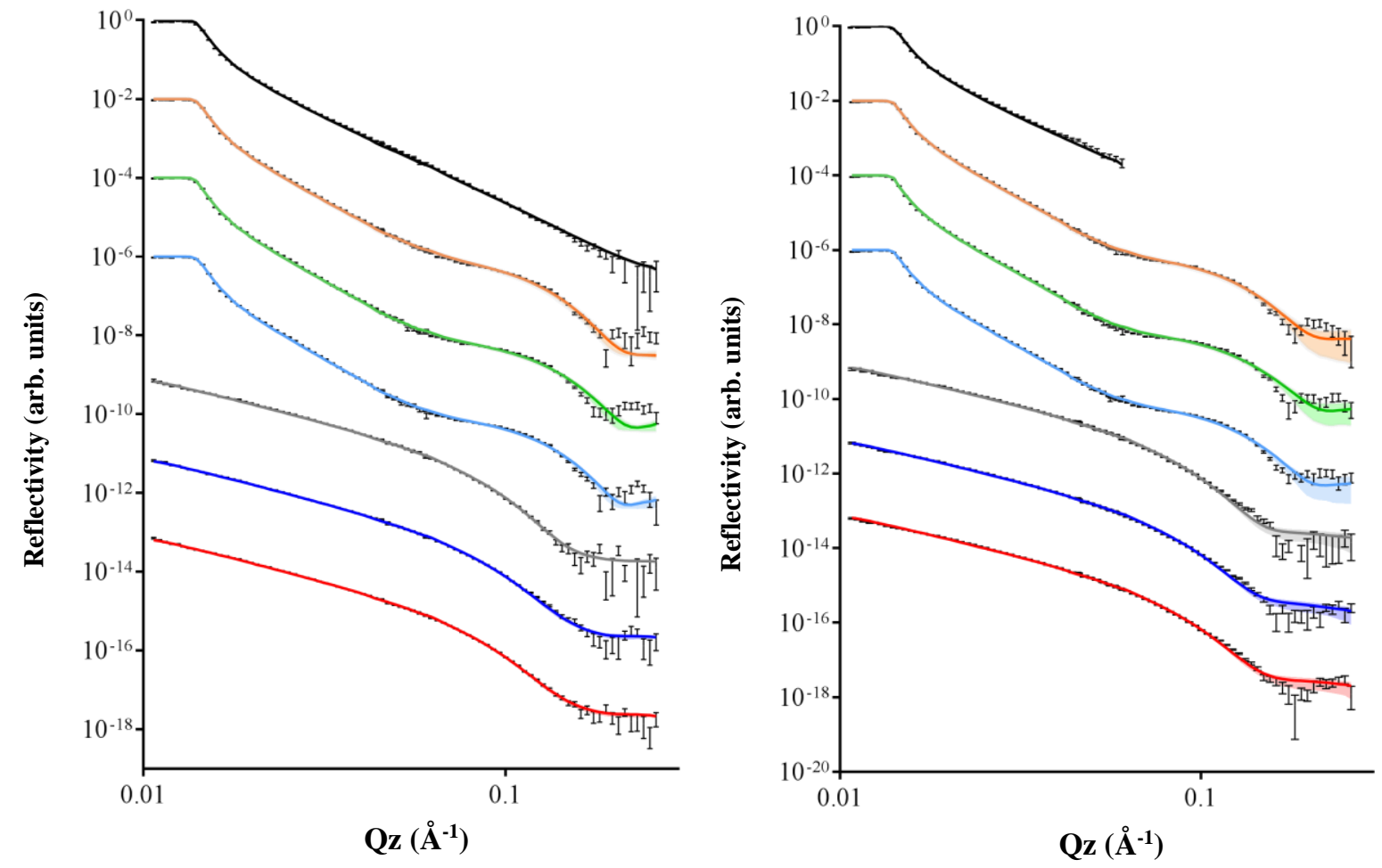

Figure S5. Reflectivity profiles for the treatment of d-POPC/cholesterol membrane mimics with (A) tolfH $(480 \mu \mathrm{M})$ or $(\mathbf{B}) \mathrm{Bi}(\text { tolf })_{3}(160 \mu \mathrm{M})$. The profiles are indicative of: plain silicon in $\mathrm{D}_{2} \mathrm{O} / \mathrm{PBS}$ (black); the d-POPC/cholesterol bilayer in $\mathrm{D}_{2} \mathrm{O} / \mathrm{PBS}$ (orange) and $\mathrm{H}_{2} \mathrm{O} / \mathrm{PBS}$ (grey); the d-POPC/cholesterol bilayer following treatment with compound in $\mathrm{D}_{2} \mathrm{O} / \mathrm{PBS}$ (green) and $\mathrm{H}_{2} \mathrm{O} / \mathrm{PBS}$ (dark blue) and; the d-POPC/cholesterol bilayer following a rinse with $\mathrm{D}_{2} \mathrm{O} / \mathrm{PBS}$ (light blue) and $\mathrm{H}_{2} \mathrm{O} / \mathrm{PBS}$ (red). Solid lines represent the best fit obtained from the data analysis and the shaded regions represent the $95 \%$ confidence intervals of the fits. Error bars represent the counting statistics (square root of the number of counts) from both the transmission and data runs propagated through the reduction process. Due to an issue with the instrument, the plain silicon in the $\mathrm{Bi}(\text { tolf })_{3}$ data set was only run at the lower incident angle $\left(0.85^{\circ}\right)$. This issue was resolved prior to the addition of the bilayer to the system, meaning data could be obtained at both incident angles $\left(0.85^{\circ}\right.$ and $\left.3.8^{\circ}\right)$ for the other datasets. 